\title{
Foreword
}

\section{QT PRODACT: Database Construction for the Evaluation of the Risk of QT Interval Prolongation by Drugs in Japan}

\author{
Haruaki Nakaya ${ }^{1}$ and Keitaro Hashimoto ${ }^{2}$
}

The main reason for the withdrawal of drugs from the market is the appearance of the side effects of prolongation of the QT interval and the resultant polymorphic ventricular tachycardia called torsade de pointes (TdP). Several structurally unrelated drugs such as terfenadine, astemizol, and cisapride have been removed from the Japanese market because of this drug-induced TdP. Recent progress in molecular biology and electrophysiology has led to a better understanding of the ionic mechanism of drug-induced QT prolongation. It is considered that drug-induced inhibition of the rapid component of the delayed rectifier $\mathrm{K}^{+}$current $\left(\mathrm{I}_{\mathrm{Kr}}\right)$, and therefore $\mathrm{hERG}$ (human ether-a-go-go related gene) channels, is the major cause of the acquired long-QT syndrome.

In order to avoid the serious side effects associated with excessive QT interval prolongation, it is important to evaluate the potential for QT interval prolongation in the process of drug development. The risk of QT interval prolongation can be assessed by non-clinical as well as clinical evaluations. For several years now, the Expert Working Group (Safety) of the International Conference on Harmonisation of Technical Requirements for Registration of Pharmaceuticals for Human Use (ICH) has been seeking a strategy to assess the potential of a test compound to delay ventricular repolarization. Guidelines for non-clinical (S7B) and clinical evaluation (E14) of QT interval prolongation and proarrhythmic potential have been developed. According to the S7B non-clinical guideline, both an in vitro $\mathrm{I}_{\mathrm{Kr}}$ assay and an in vivo QT assay are recommended as a standard non-clinical testing strategy to assess the potential risk of QT interval prolongation.

Members representing the Japan Pharmaceutical Manufactures Association (JPMA) participated in the meeting of the Expert Working Group (Safety) of ICH and took part in drafting the final guidelines. In the course of discussion, they realized the necessity of constructing databases for a more accurate and more reliable evaluation of the risk of QT interval prolongation. Accordingly, the members from pharmaceutical companies belonging to the JPMA and contract laboratories belonging to the Japan Association of Contract Laboratories for Safety Evaluation (JACL) designed a project named "QT Interval Prolongation: Project for Database Construction (QT PRODACT)". The QT PRODACT consists of in vitro action potential assays and in vivo QT assays. The most sensitive in vitro assay to evaluate the risk of QT prolongation may be patch clamp experiments using cultured cells expressing hERG channels or isolated native cardiomyocytes. However, the in vitro action potential assay using guinea-pig papillary muscles, described in this issue, appears to provide useful and reliable information about the risk of QT prolongation when the differences of action potential durations at two repolarization levels are measured. Such an in vitro action potential assay to test triangulation of the action potential would be a convenient method for a preliminary test in place of the hERG channel assay, although the in vitro action potential assay would be included in follow-up non-clinical studies according to the ICH S7B guideline. In vivo QT assays using conscious dogs, conscious monkeys, and anesthetized dogs also afford useful information about the most appropriate in vivo model.

Both assays (in vitro action potential assays and in vivo QT assays) were conducted to test many positive and negative compounds in many laboratories of JPMA and JACL. The data were accumulated, analyzed, and summarized as databases. The QT PRODACT database is now published in this special issue of the Journal of Pharmacological Sciences. We hope the articles on QT PRODACT published in this issue will provide useful information for accurate evaluation of the risk of QT interval prolongation in safety pharmacology.

\footnotetext{
${ }^{1}$ Department of Pharmacology, Chiba University Graduate School of Medicine, Chiba 260-8670, Japan nakaya@faculty.chiba-u.jp

${ }^{2}$ Department of Pharmacology, Interdisciplinary Graduate School of Medicine and Engineering, University of Yamanashi, Yamanashi 409-3898, Japan keitaroh@yamanashi.ac.jp
} 